\title{
Interaction between miRNAs and IncRNAs in Breast Cancer with Special Focus on Exosomal Derived miRNAs in Breast Cancer
}

\author{
Kaifee ARMAN $^{1}$, Zekiye ALTAN $^{1}$, Yunus SAHIN ${ }^{1}$, Yusuf Ziya IGCI ${ }^{1}$ \\ ${ }^{1}$ Department of Medical Biology, Faculty of Medicine, Gaziantep University, Şahinbey, \\ Gaziantep,Turkey \\ *kaifeearman786@gmail.com
}

\begin{abstract}
Breast cancer is a matter of great concern as it is the most common life-threatening diseases for women.Non-coding RNAs has a pivotal role in breast cancer. These non-coding RNAs, for example microRNAs and long non-coding RNAs are vital regulatory molecules which play critical role in breast cancer. In this review, we discuss about the interaction between IncRNA and miRNAs in breast cancer. Apart from this, we discuss exosomal miRNAs involved in breast cancer. These exosomal miRNAs are known for their use as biomarkers. They can also be used as agents for delivering antitumor drugs. This revew compiles all the data related to IncRNA:miRNA interaction and exosomal miRNAs involved in breast cancer which can be exploited as therapeutic mechanism as well as to modulate the tumor microenvironment for breast cancer diagnosis and treatment.
\end{abstract}

Keywords: breast cancer, IncRNA, miRNA, exosome

\section{INTRODUCTION}

Breast cancer is known to be the most frequently occuring malignant disease among females. At the same time, it ranks second for mortality among all cancers in women [1]. In recent past, there is more survival rate while treating breast cancer patients [2], but completely understanding the molecular mechanism in treating a breast cancer is still a major challenge ahead of us. Thus it is the urge of time to concentrate on molecular mechanisms for putting a final check on breast cancer.

The earlier point of view regarding gene expression pattern has completely changed after 'non coding revolution'. Recent researches are concentrating to exploit this non coding revolution for treating many cancers including breast cancer. It has been shown that non-coding RNAs (ncRNAs) including small $(<200 \mathrm{~kb})$ and long (lncRNAs) $(>200 \mathrm{~kb}$ ), are involved in both tumor suppressive and oncogenic pathways [3-6]. Among these ncRNAS, miRNA and lncRNA play an important role in regulating gene expression in many cancers. miRNAs are known to be short regulatory RNAs, approximately 19-24 nucleotides in length and regulate gene expression at the post-transcriptional level. These are transcribed mainly by RNA polymerase II or III as pri-miRNAs, which are processed and further cleaved into pre-miRNAs in the nucleus by Drosha-DGCR8[7,8]. Then the cleaved portion of the hairpin (premicroRNA) is exported to the cytoplasm to complete its processing by the cytoplasmic RNAse III enzyme Dicer[9]. The mature miRNA modulates gene expression by binding to partially complementary sequences in the 3'UTRs or 5'UTRs of target mRNAs leading to translational inhibition or degradation of the target mRNA[10]. miRNAs are known to play crucial roles in the development of tumor and are known to be differentially expressed in different molecular subtypes of tumors. The classification of tumor malignancies by individual miRNAs is based on their expression profiles $[11,12]$. The amplification of oncogenic miRNAs containing chromosomal regions is linked to cancer development and their upregulation silences the tumor suppressor genes[13]. Whereas when we look at the tumor-suppressive miRNAs, they are often located in chromosomal fragile sites; and loss of their expression and finally upregulation of their target oncogenes takes place due to deletion or mutation of these regions [14]. Several miRNAs have been identified in breast cancer with their role as tumor suppressors or oncogenes and known to be regulators of tumor initiation, metastasis and chemoresistance[15].

Long ncRNAs are defined as any RNA molecule or transcript with no coding potential and a size greater than 200 nucleotides [16-18]. The biogenesis of IncRNAs are similar to those of mRNAs 
employing the same transcriptional machinery (Pol II), and involving almost same types of epigenetic regulation such as histone-modification profiles and splicing signals[17, 19, 20]. Atleast $50 \%$ of lncRNAs are also capped and polyadenylated. The expression of lncRNA is highly cell-type specific, but to a lesser extent as compared to protein-coding genes[21-23].

\section{INTERACTION BETWEEN MIRNAS AND InCRNAS}

Recently it has been found that long non-coding RNA (lncRNA) transcribed from an ultraconserved region mediates the post-transcriptional control of microRNAs at the level of Drosha processing [24].IncRNAs have an intrinsic ability to interact with DNA, RNA andproteins. They act as guides, tethers, decoys and scaffolds and this explain their ability to regulate gene expression [25, 26]. Moreover, many recent studies have shown connections between microRNAs and lncRNA. A new concept of competing endogenous RNAs (ceRNAs) was proposed in order to explain a novel regulatory mechanism of RNA. This new concept states that RNAs cancross-talk with each other imposing different level of post-transcriptional regulation [27]. This mechanism was first of all seen in breast cancer. Many studies have shown that some lncRNAs are under the regulation of microRNAs to reducetheir stability. It is the ability of numerous lncRNA transcripts to compete for microRNA binding, thereby alleviating the negative effect of microRNAs on their respective mRNA targets[28]. One of the way through which most lncRNA communicate with miRNA is microRNA Response Elements (MREs).

As miRNAs and lncRNAs are in abundance, miRNA:IncRNAs interaction should be universal and functionally vital. In many cases lncRNAs harbor the recognition site for functional miRNAs titrating the miRNAs from their mRNA targets. IncRNAs themselves could be targeted by miRNAs.IncRNAs also compete with miRNAs for binding the same mRNAs, the competition would block the recognition of miRNAs and thus increase mRNA translation [29]. This interaction is seen in many cancers. Their interaction in breast cancer has very important role in deciding the prospect of breast cancer.

Similarly, lncRNA LOC554202 could encode miR-31, and promoter methylation of lncRNA LOC554202 leads to decreased miR-31 and thus contributes to breast cancer invasion and metastasis [30]. In a recent study, Hou et al. showed interaction between linc-RoR and miR-205. Linc-RoR functions as a microRNA sponge for miR-205. Eventually, downregulation of miR-205 target genes such as ZEB2 is prevented by linc-RoR, that results in EMT and metastasis in breast cancer[31]. In another study, direct interaction between lncRNA Urothelial Carcinoma Associated 1(UCA1) and miR-143 in breast cancer is shown. Infact UCA1 down regulates miR-143 and modulates breast cancer cell growth and apoptosis [32]. There is another interesting study that shows that lncRNA ATB competitively binds to miR-200c due to three putative bindig regions for miR-200 family and then increases trastuzumab resistance and invasion-metastasis cascade in breast cancer which finally leads to upregulation of ZEB1 and ZNF-217 inducing EMT [33, 34]. LincRNA HOTAIR inhibits miR-7 indirectly in breast cancer stem cells which directly inhibits SETDB1 and reverses the EMT of breast cancer stem cells by downregulating the STAT3 pathway[35]. A negative correlation between miR-21 and GAS5 has also been found in breast tumor specimens.GAS5 is downregulated and miR-21 is upregulated in the breast tumors[36].

\section{EXOSOMAL miRnAS INVOLVED IN BREAST CANCER}

Exosomes are nanovesicles secreted into the extracellular environment upon internal vesicle fusion with the plasma membrane. Exosomes are gaining importance as one of the key players in the crosstalk between cancer and its microenvironment. Evidences suggest that exosomes may be used as biomarkers for the diagnosis and prognosis of malignant tumors[37]. miRNAs are found to be secreted in the exosomes and can modulate the function of receptor cells.Exosomal miRNAs are known to be implicated in several aspects of breast tumor development[38, 39].Exosome-derived miRNA are known to mediate intercellular communication between tumor-associated macrophages and breast cancer cells. In a study, it has been demonstrated that miR-223 containing exosomes released from macrophages, enter breast cancer cells and promote cell migration and invasion [39]. miR-122 secreted extracellular vesicles within breast cancer and was shown to dysregulate nutrient metabolism by downregulating pyruvate kinase [40]. Exosomes released from mesenchymal stem cells contained miR-16, resulting in a decrease in VEGF expression in breast cancer cells [41].Another exosomal miRNAs from bone marrow mesenchymal stem cells like miR-23b promotes 
dormancy in metastatic breast cancer cells [42]. miR-21 and 34a are other examples of bone marrow derived exosomes which move to breast cancer cells and has pro-tumor and anti-apoptotic effects [4345]. There are other exosomal miRNAs like miR-127, miR-197, miR-222 and miR-223 originating from bone marrow stroma inhibiting breast cancer cell proliferation by targeting CXCL12 chemokine gene [38]. Zhou et al.showed that miR-105is a potent regulator of migration targeting the tight junction protein ZO-1 and thus promoting the metastasis of breast cancer[46]. In a recent study, Chen et al. Showed that exosomal miRNAs like miR-17, miR-30a, miR-100, and miR-222 which are released from drug-resistant breast cancer cells may spread resistance capacity to sensitive ones[47]. In an another study, it is shown that breast tumor cells can release exosomes containing miR-221/222, which play an important role in resistance to tamoxifen, an estrogen receptor antagonist used against particular types of breast tumor [48].

\section{Conclusion}

In this review, we focus on the interplay between lncRNAs and microRNAs in breast cancer. Here we try to summarize various examples in order to describe lncRNA:microRNA interactions in breast cancer. These two types of ncRNAs act in a synchronised manner to regulate the gene expression. This study will help in better understanding the interaction between miRNA and lncRNA in breast cancer in a concised way. Besides the IncRNA:miRNA interaction in breast cancer, exosomal miRNAs involved in breast cancer is also discussed. These exosomal miRNAs have their use as biomarkers in breast cancer. They may also be used as agents for delivering antitumor drugs. These compiled data can be exploited as therapeutic mechanism and also to modulate the tumor microenvironment for breast cancer diagnosis and treatment.

\section{ACKNOWLEDGEMENT}

Kaifee Arman is recipient of Graduate Scholarship from Turkish Scientific and Technological Research Council (TUBITAK) under the program 2215- Graduate Scholarship Programme for International Students.

\section{REFERENCES}

[1] Siegel, R., et al., Cancer statistics, 2014. CA Cancer J Clin, 2014. 64(1): p. 9-29.

[2] DeSantis, C.E., et al., Cancer treatment and survivorship statistics, 2014. CA Cancer J Clin, 2014. 64(4): p. 252-71.

[3] Malone, C.D. and G.J. Hannon, Small RNAs as guardians of the genome. Cell, 2009. 136(4): p. 656-68.

[4] Moazed, D., Small RNAs in transcriptional gene silencing and genome defence. Nature, 2009. 457(7228): p. 413-20.

[5] Brosnan, C.A. and O. Voinnet, The long and the short of noncoding RNAs. Curr Opin Cell Biol, 2009. 21(3): p. 416-25.

[6] Mattick, J.S., Non-coding RNAs: the architects of eukaryotic complexity. EMBO Rep, 2001. 2(11): p. 986-91.

[7] Lee, Y., et al., The nuclear RNase III Drosha initiates microRNA processing. Nature, 2003. 425(6956): p. 415-9.

[8] Denli, A.M., et al., Processing of primary microRNAs by the Microprocessor complex. Nature, 2004. 432(7014): p. 231-5.

[9] Hutvagner, G., et al., A cellular function for the RNA-interference enzyme Dicer in the maturation of the let-7 small temporal RNA. Science, 2001. 293(5531): p. 834-8.

[10] Eulalio, A., E. Huntzinger, and E. Izaurralde, GW182 interaction with Argonaute is essential for miRNA-mediated translational repression and mRNA decay. Nat Struct Mol Biol, 2008. 15(4): p. 346-53.

[11] Blenkiron, C., et al., MicroRNA expression profiling of human breast cancer identifies new markers of tumor subtype. Genome Biol, 2007. 8(10): p. R214.

[12] Iorio, M.V., et al., MicroRNA gene expression deregulation in human breast cancer. Cancer Res, 2005. 65(16): p. 7065-70. 
[13] He, L., et al., A microRNA polycistron as a potential human oncogene. Nature, 2005. 435(7043): p. 828-33.

[14] Yamamoto, Y., et al., An integrative genomic analysis revealed the relevance of microRNA and gene expression for drug-resistance in human breast cancer cells. Mol Cancer, 2011. 10: p. 135.

[15] Takahashi, R.U., H. Miyazaki, and T. Ochiya, The Roles of MicroRNAs in Breast Cancer. Cancers (Basel), 2015. 7(2): p. 598-616.

[16] Harrow, J., et al., GENCODE: the reference human genome annotation for The ENCODE Project. Genome Res, 2012. 22(9): p. 1760-74.

[17] Derrien, T., et al., The GENCODE v7 catalog of human long noncoding RNAs: analysis of their gene structure, evolution, and expression. Genome Res, 2012. 22(9): p. 1775-89.

[18] Guttman, M., et al., Ribosome profiling provides evidence that large noncoding RNAs do not encode proteins. Cell, 2013. 154(1): p. 240-51.

[19] Guttman, M., et al., Chromatin signature reveals over a thousand highly conserved large noncoding RNAs in mammals. Nature, 2009. 458(7235): p. 223-7.

[20] Tordonato, C., P.P. Di Fiore, and F. Nicassio, The role of non-coding RNAs in the regulation of stem cells and progenitors in the normal mammary gland and in breast tumors. Front Genet, 2015. 6: p. 72.

[21] Cabili, M.N., et al., Integrative annotation of human large intergenic noncoding RNAs reveals global properties and specific subclasses. Genes Dev, 2011. 25(18): p. 1915-27.

[22] Djebali, S., et al., Landscape of transcription in human cells. Nature, 2012. 489(7414): p. 101-8.

[23] Ravasi, T., et al., Experimental validation of the regulated expression of large numbers of noncoding RNAs from the mouse genome. Genome Res, 2006. 16(1): p. 11-9.

[24] Liz, J., et al., Regulation of pri-miRNA processing by a long noncoding RNA transcribed from an ultraconserved region. Mol Cell, 2014. 55(1): p. 138-47.

[25] Brockdorff, N., Noncoding RNA and Polycomb recruitment. RNA, 2013. 19(4): p. 429-42.

[26] Gong, C. and L.E. Maquat, lncRNAs transactivate STAU1-mediated mRNA decay by duplexing with 3' UTRs via Alu elements. Nature, 2011. 470(7333): p. 284-8.

[27] Hansen, T.B., et al., Natural RNA circles function as efficient microRNA sponges. Nature, 2013. 495(7441): p. 384-8.

[28] Liz, J. and M. Esteller, lncRNAs and microRNAs with a role in cancer development. Biochim Biophys Acta, 2015.

[29] Yang, G., X. Lu, and L. Yuan, LncRNA: a link between RNA and cancer. Biochim Biophys Acta, 2014. 1839(11): p. 1097-109.

[30] Augoff, K., et al., miR-31 and its host gene lncRNA LOC554202 are regulated by promoter hypermethylation in triple-negative breast cancer. Mol Cancer, 2012. 11: p. 5.

[31] Hou, P., et al., LincRNA-ROR induces epithelial-to-mesenchymal transition and contributes to breast cancer tumorigenesis and metastasis. Cell Death Dis, 2014. 5: p. e1287.

[32] Tuo, Y.L., X.M. Li, and J. Luo, Long noncoding RNA UCA1 modulates breast cancer cell growth and apoptosis through decreasing tumor suppressive miR-143. Eur Rev Med Pharmacol Sci, 2015. 19(18): p. 3403-11.

[33] Shi, S.J., et al., LncRNA-ATB promotes trastuzumab resistance and invasion-metastasis cascade in breast cancer. Oncotarget, 2015. 6(13): p. 11652-63.

[34] Yuan, J.H., et al., A long noncoding RNA activated by TGF-beta promotes the invasionmetastasis cascade in hepatocellular carcinoma. Cancer Cell, 2014. 25(5): p. 666-81.

[35] Zhang, H., et al., MiR-7, inhibited indirectly by lincRNA HOTAIR, directly inhibits SETDB1 and reverses the EMT of breast cancer stem cells by downregulating the STAT3 pathway. Stem Cells, 2014. 32(11): p. 2858-68.

[36] Zhang, Z., et al., Negative regulation of lncRNA GAS5 by miR-21. Cell Death Differ, 2013. 20(11): p. 1558-68.

[37] Properzi, F., M. Logozzi, and S. Fais, Exosomes: the future of biomarkers in medicine. Biomark Med, 2013. 7(5): p. 769-78. 
[38] Lim, P.K., et al., Gap junction-mediated import of microRNA from bone marrow stromal cells can elicit cell cycle quiescence in breast cancer cells. Cancer Res, 2011. 71(5): p. 1550-60.

[39] Yang, M., et al., Microvesicles secreted by macrophages shuttle invasion-potentiating microRNAs into breast cancer cells. Mol Cancer, 2011. 10: p. 117.

[40] Kosaka, N., et al., Neutral sphingomyelinase 2 (nSMase2)-dependent exosomal transfer of angiogenic microRNAs regulate cancer cell metastasis. J Biol Chem, 2013. 288(15): p. 1084959.

[41] Lee, J.K., et al., Exosomes derived from mesenchymal stem cells suppress angiogenesis by down-regulating VEGF expression in breast cancer cells. PLoS One, 2013. 8(12): p. e84256.

[42] Ono, M., et al., Exosomes from bone marrow mesenchymal stem cells contain a microRNA that promotes dormancy in metastatic breast cancer cells. Sci Signal, 2014. 7(332): p. ra63.

[43] Baglio, S.R., et al., Human bone marrow- and adipose-mesenchymal stem cells secrete exosomes enriched in distinctive miRNA and tRNA species. Stem Cell Res Ther, 2015. 6: p. 127.

[44] Vallabhaneni, K.C., et al., Extracellular vesicles from bone marrow mesenchymal stem/stromal cells transport tumor regulatory microRNA, proteins, and metabolites. Oncotarget, 2015. 6(7): p. 4953-67.

[45] Frankel, L.B., et al., Programmed cell death 4 (PDCD4) is an important functional target of the microRNA miR-21 in breast cancer cells. J Biol Chem, 2008. 283(2): p. 1026-33.

[46] Zhou, W., et al., Cancer-secreted miR-105 destroys vascular endothelial barriers to promote metastasis. Cancer Cell, 2014. 25(4): p. 501-15.

[47] Chen, W.X., et al., Exosomes from drug-resistant breast cancer cells transmit chemoresistance by a horizontal transfer of microRNAs. PLoS One, 2014. 9(4): p. e95240.

[48] Wei, Y., et al., Exosomal miR-221/222 enhances tamoxifen resistance in recipient ER-positive breast cancer cells. Breast Cancer Res Treat, 2014. 147(2): p. 423-31. 